\title{
Connecting Our Gut Feeling and How Our Gut Feels: The Role of Well-being Attributes in Irritable Bowel Syndrome
}

\author{
Ashkan Farhadi, ${ }^{1 *}$ Dwaine Banton, ${ }^{2}$ and Laurie Keefer ${ }^{3}$ \\ ${ }^{I}$ Section of Gastroenterology and Nutrition, University of California, Irvine, CA, USA; ${ }^{2}$ Janssen Research and Development, Spring House, PA, \\ USA; and ${ }^{3}$ Icahn School of Medicine at Mount Sinai, Division of Gastroenterology, New York, NY, USA
}

\section{Background/Aims}

There is a close relationship between the mind and gut in the pathogenesis of functional bowel disorders. Common psychological disturbances such as depression and anxiety are not uncommon in those with irritable bowel syndrome (IBS). There is little research investigating the role of positive psychology and gastrointestinal (GI) conditions. In this pilot study we investigated the well-being attributes in those with and without IBS.

\section{Methods}

We used an anonymous online survey and recruited 416 study subjects using social media as the main method of recruitment. We gathered demographic information, Gl symptoms, history of mental health issues such as anxiety and depression, assessed several well-being attributes, and finally assessed subjective well-being. We hypothesized that those with GI symptoms and IBS have lower scores in their well-being attributes compared to healthy controls.

\section{Results}

We observed that a history of anxiety and depression is significantly associated with GI symptoms and IBS. In addition, sense of subjective well-being and several well-being attributes are negatively associated with GI symptoms and/or IBS. Of interest, the household income showed a negative correlation with the prevalence of GI symptoms and IBS.

\section{Conclusions}

Subjective well-being, and several well-being attributes that contribute to the sense of overall contentment, are negatively associated with GI symptoms and IBS. The link between subjective well-being, and GI symptoms and IBS are independent of anxiety and depression. Well-being attributes and sense of subjective well-being may be a contributory factor in clinical expression of $\mathrm{Gl}$ symptoms or IBS consistent with the biopsychosocial model of the disease.

(J Neurogastroenterol Motil 2018;24:289-298)

\section{Key Words}

Anxiety; Depression; Happiness; Irritable bowel syndrome; Subjective well-being

Received: September 19, 2017 Revised: November 23, 2017 Accepted: January 21, 2018

(c) This is an Open Access article distributed under the terms of the Creative Commons Attribution Non-Commercial License (http://creativecommons. org/licenses/by-nc/4.0) which permits unrestricted non-commercial use, distribution, and reproduction in any medium, provided the original work is properly cited.

*Correspondence: Ashkan Farhadi, MD, MS, FACP, FACG

Memorial Care Medical Group, 722 Baker St. Costa Mesa, Irvine, CA 92626, USA

Tel: +1-714-966 9506, Fax: +1-714-966 9567, E-mail: ashkan_farhadi@lhavelBS.com 


\section{Introduction}

Irritable Bowel Syndrome (IBS) is one of the most common gastrointestinal (GI) disorders, characterized by recurring abdominal pain and changes in bowel habits. The prevalence of this disorder is estimated to be $10-25 \%$ in different populations. ${ }^{1,2}$ The etiology of IBS is not known, and several biological, psychological, and sociological factors are thought to be involved in the pathogenesis and natural history of this condition in a biopsychosocial model. ${ }^{3}$ There is no question that there is a close connection between our gut and brain in the form of brain-gut axis, and this close connection has a pivotal role in the pathogenesis of GI symptoms in the form of functional gastrointestinal disorders (FGID) and in particular IBS. Based on the biopsychosocial model, genetic factors and early life experiences as well as later life events such as abuse history or life stress are key elements in the way our bodies adapt to situations and cope with the different conditions. These factors are the basis for development of many of our personality traits and/or progression of mental health issues later in life. ${ }^{3}$ Several studies have shown that emotional stress and psychological factors are closely connected to GI symptoms and pathogenesis of IBS. ${ }^{4-9}$ This may explain why based on Rome IV, it has been proposed to use the new terminology of Disorders of Gut-Brain Interaction instead of FGID. ${ }^{10}$ There are several studies that have already explored the role of commonly diagnosed conditions such as anxiety and depression in FGID and IBS. ${ }^{11-16}$ In addition, some studies explored personality traits in IBS and general digestive disease. ${ }^{17-19}$

Several studies on IBS have shown that quality of life is significantly affected in a negative way. ${ }^{20-23}$ Subjective well-being and happiness, on the other hand, are closely associated with better mental health and overall well-being. ${ }^{24,25}$ Subjective well-being along with other measures of quality of life are negatively affected, in those who suffer from chronic recurring illness such as IBS. Some studies showed that the general sense of well-being is disturbed in those with IBS-like symptoms who suffered microscopic colitis. ${ }^{26}$ However, whether subjective well-being and life satisfaction are negatively affected in IBS, independent of anxiety and depression, has not been extensively explored.

Positive psychology provides a model that focuses on factors that influence resilience, or one's ability to recover from adversity such as symptoms of IBS. There are biological underpinnings or strengths that could counteract the negative impact of pain or stress on the body (allostatic load), such as in IBS. Understanding the extent to which core human strengths or well-being attributes, such as optimism, confidence, trust, and sense of financial security, could help in the creation of stronger coping mechanism or broader social support for IBS needs to be explored. How these well-being attributes could contribute to IBS and its symptomology may provide a new framework of either prevention or early intervention (eg, building resilience or strengths). In this study, we carried out an online survey to assess the relationship of factors that determine subjective well-being with GI symptoms and IBS. Our aim was to determine if subjective well-being or and other well-being attributes relate to GI symptoms or IBS compared to controls. Also we evaluated whether these attributes relate to IBS independent of IBS comorbid conditions such as anxiety and depression.

\section{Materials and Methods}

\section{Subjects and Recruitment}

The study was approved by Memorial Care foundation Institutional Review Board (IRB No. MHS\#731-17). All authors had access to the study data and had reviewed and approved the final manuscript. Individuals ( $n=416 / 421$ completed surveys) were recruited for this study mainly through Facebook posting and Facebook advertising. The majority of the cases (92\%) lived in the United States. We asked the participants to fill an online questionnaire using the Survey Monkey (Table). The questionnaire was devoid of any identifying questions to ensure participant anonymity. The participants were adults more than 18 years old.

\section{Questionnaire}

There were several questions exploring demographic information, including age, gender, level of education, occupation, household income, household number and country of residence. The GI questions were mainly generalized questions regarding presence and severity of abdominal pain and changes in bowel habit, including diarrhea and constipation. We were able to determine if the individuals were satisfying the criteria of IBS based on the Rome IV criteria: abdominal pain or changes in bowel habit that bother them more than once a week for the last 3 months. We asked questions to assess the presence of self-reported anxiety and depression. We also used validated Generalized Anxiety Disorder 7-item Scale (GAD7) and Patient Health Questionnaire 9-item Scale (PHQ-9), which are self administered screening tools for evaluation of current status of anxiety and depression, respectively. Finally, we used a Positive Psychology Questionnaire generated by the authors that assessed the subjective and several well-being attributes presented in Table. 
Table. The Average Score of the Well-being Attributes in Those With and Without Gastrointestinal Symptoms and Irritable Bowel Syndrome

\begin{tabular}{|c|c|c|c|c|c|c|}
\hline Well-being attribute & $\begin{array}{c}\text { Healthy } \\
\text { control } \\
(n=191)\end{array}$ & $\begin{array}{l}\text { GI symptoms } \\
\text { without IBS } \\
(\mathrm{n}=134)\end{array}$ & $\begin{array}{c}\text { IBS } \\
(\mathrm{n}=91)\end{array}$ & $\begin{array}{c}\text { Control vs GI } \\
\text { symptoms } \\
\text { without IBS }\end{array}$ & $\begin{array}{c}\text { IBS vs GI } \\
\text { symptoms } \\
\text { without IBS }\end{array}$ & $\begin{array}{l}\text { Controls } \\
\text { vs IBS }\end{array}$ \\
\hline Flexibility & 3.68 & 3.66 & 3.74 & $P=0.852$ & $P=0.525$ & $P=0.613$ \\
\hline Confidence & 3.60 & $3.29^{\mathrm{a}}$ & $3.27^{\mathrm{a}}$ & $P=0.006$ & $P=0.865$ & $P=0.011$ \\
\hline Sense of financial security & 3.07 & $2.72^{\mathrm{a}}$ & $2.24^{\mathrm{a}, \mathrm{b}}$ & $P=0.010$ & $P=0.008$ & $P<0.001$ \\
\hline Feeling loved & 4.05 & $3.83^{\mathrm{a}}$ & $3.55^{\mathrm{a}}$ & $P=0.034$ & $P=0.067$ & $P<0.001$ \\
\hline Having someone to love & 4.10 & 4.06 & 3.95 & $P=0.687$ & $P=0.442$ & $P=0.233$ \\
\hline Feeling healthy and fit & 3.33 & $2.88^{\mathrm{a}}$ & $2.31^{\mathrm{a}, \mathrm{b}}$ & $P<0.001$ & $P<0.001$ & $P<0.001$ \\
\hline Believe in faith & 3.02 & 3.21 & 3.05 & $P=0.218$ & $P=0.403$ & $P=0.858$ \\
\hline Being generous & 3.24 & $2.95^{\mathrm{a}}$ & 3.18 & $P=0.019$ & $P=0.149$ & $P=0.664$ \\
\hline Having a passion in life & 3.39 & $3.15^{\mathrm{a}}$ & 3.18 & $P=0.048$ & $P=0.827$ & $P=0.144$ \\
\hline Being supportive of others & 4.11 & 4.10 & 4.29 & $P=0.944$ & $P=0.079$ & $P=0.077$ \\
\hline Having a desirable job & 4.12 & $3.87^{\mathrm{a}}$ & $3.51^{\mathrm{a}, \mathrm{b}}$ & $P=0.012$ & $P=0.011$ & $P<0.001$ \\
\hline Sense of independence & 3.98 & 3.79 & $3.55^{\mathrm{a}}$ & $P=0.109$ & $P=0.142$ & $P=0.001$ \\
\hline Having adequate education & 3.89 & 3.75 & $3.63^{\mathrm{a}}$ & $P=0.158$ & $P=0.391$ & $P=0.033$ \\
\hline Having adequate leisure & 3.61 & 3.58 & 3.49 & $P=0.757$ & $P=0.487$ & $P=0.315$ \\
\hline Being respected & 3.99 & $3.71^{\mathrm{a}}$ & $3.35^{\mathrm{a}, \mathrm{b}}$ & $P=0.002$ & $P=0.006$ & $P<0.001$ \\
\hline Feeling their talent is being properly used & 3.82 & $3.53^{\mathrm{a}}$ & $3.22^{\mathrm{a}, \mathrm{b}}$ & $P=0.002$ & $P=0.017$ & $P<0.001$ \\
\hline Having adequate pleasure & 3.51 & 3.36 & $3.04^{\mathrm{a}, \mathrm{b}}$ & $P=0.108$ & $P=0.007$ & $P<0.001$ \\
\hline Being attractive & 3.43 & 3.45 & $3.13^{\mathrm{a}, \mathrm{b}}$ & $P=0.868$ & $P=0.008$ & $P=0.004$ \\
\hline Being forgiving & 3.77 & 3.75 & 3.56 & $P=0.822$ & $P=0.195$ & $P=0.093$ \\
\hline Feeling accepted by others & 4.11 & $3.93^{\mathrm{a}}$ & $3.69^{\mathrm{a}, \mathrm{b}}$ & $P=0.038$ & $P=0.046$ & $P<0.001$ \\
\hline Living in peace & 3.76 & $3.53^{\mathrm{a}}$ & $3.28^{\mathrm{a}, \mathrm{b}}$ & $P=0.014$ & $P=0.042$ & $P<0.001$ \\
\hline Managing life properly & 3.57 & $3.31^{\mathrm{a}}$ & $3.09^{\mathrm{a}}$ & $P=0.008$ & $P=0.083$ & $P<0.001$ \\
\hline Being optimistic & 3.83 & 3.72 & $3.50^{\mathrm{a}}$ & $P=0.208$ & $P=0.088$ & $P=0.004$ \\
\hline Being able to trust others & 3.89 & 3.80 & $3.60^{\mathrm{a}}$ & $P=0.400$ & $P=0.154$ & $P=0.023$ \\
\hline Believe in fairness in life & 3.21 & 3.19 & $2.85^{\mathrm{a}, \mathrm{b}}$ & $P=0.828$ & $P=0.017$ & $P=0.003$ \\
\hline Being grateful & 4.26 & 4.33 & 4.17 & $P=0.427$ & $P=0.156$ & $P=0.409$ \\
\hline Embracing challenges in life & 3.62 & 3.44 & 3.49 & $P=0.078$ & $P=0.706$ & $P=0.265$ \\
\hline Being successful & 3.77 & $3.52^{\mathrm{a}}$ & $3.12^{\mathrm{a}, \mathrm{b}}$ & $P=0.008$ & $P=0.006$ & $P<0.001$ \\
\hline Excess regret from the past & 3.43 & 3.23 & $3.14^{\mathrm{a}}$ & $P=0.052$ & $P=0.513$ & $P=0.024$ \\
\hline Feeling closure with death & 2.96 & 3.16 & 3.21 & $P=0.283$ & $P=0.841$ & $P=0.255$ \\
\hline Overall happiness with life & 3.88 & $3.72^{\mathrm{a}}$ & $3.31^{\mathrm{a}, \mathrm{b}}$ & $P=0.045$ & $P=0.001$ & $P<0.001$ \\
\hline
\end{tabular}

${ }^{a} \mathrm{~A}$ significant difference compared with controls.

${ }^{\mathrm{b}}$ A significant difference in those with gastrointestinal (GI) symptoms with or without irritable bowel syndrome (IBS).

The face validity of the questions was established by 2 experts. The test was pilot tested on a subset of participants for practicality and feasibility of the questionnaire. We intentionally posed some questions with overlapping theme for testing for internal consistency. The Cronbach's alpha for these questions were 0.64-0.71, indicating good internal consistency in the responses. The participants were asked to rank the provided choices that were also graded from 1 (low) to 5 (high) based on their perceived importance.

\section{Missing Data Management}

We attempted to minimize missing data by planning well the study to minimize asking too many detailed questions, to increase the chance of having a complete response. For example, If someone did not have any issue with anxiety they would have bypassed the anxiety related questions altogether. We had on average $13.9 \%$ of well-being attribute questions that were left unanswered. This number was $13.8 \%$ in those without IBS and $14.3 \%$ in those with IBS, which suggests a "missing at random" assumption. Considering this assumption we used a list-wise deletion for handling our missing values. 

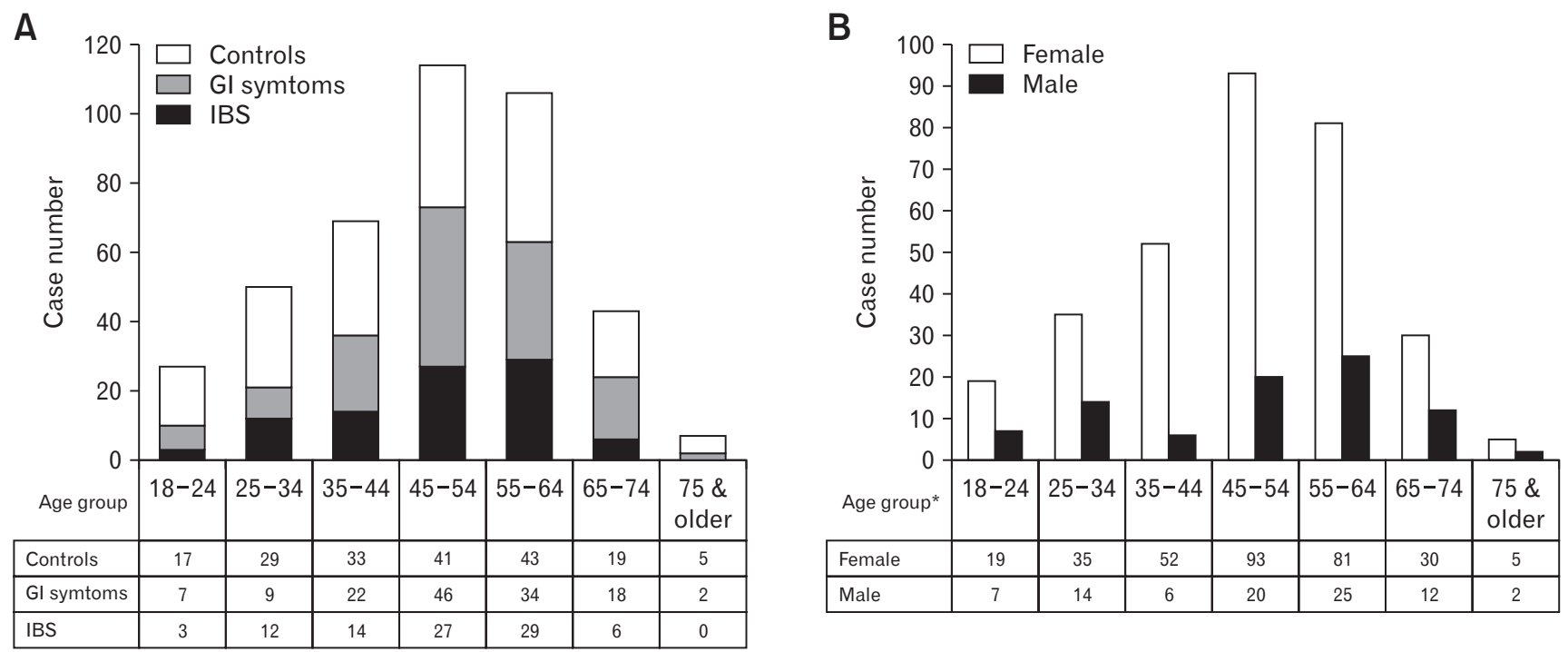

Figure 1. The age and gender distribution of the subjects with and without gastrointestinal (GI) symptoms and irritable bowel syndrome (IBS). (A) The age distribution of our subjects. (B) The gender distribution of our subjects. *Data from only 401 participants who completed both age and gender information in our study.

\section{Statistical Methods}

The data were exported into an Excel spreadsheet. Presence of GI symptoms was based on a question with yes/no answer but the presence of IBS was based on a variable that was generated based on 2 other questions that determined the frequency of abdominal pain episodes and frequency of abnormal bowel movement. Both were measured as dichotomous variables. We compared healthy controls versus those with GI symptoms without IBS to those with IBS as main groups. We used SPSS version 23 software (IBM, New York, USA) for analysis of the data. For direct comparison between 2 variables, the Mann-Whitney $U$ test, chi-squared test of independence, or a $t$ test of independence were used as appropriate. The main statistical tools that were used to characterize the relationship among various variables of interest were Kruskal-Wallis test and binary logistic regression. For this study, a $P$-value $<0.05$ was considered significant. Due to exploratory nature of our study, we did not use Bonferroni adjustment to be able to pinpoint potential correlations that could be established in the future confirmatory studies with larger subject numbers.

\section{Results}

The majority of the participants were young to middle aged adults in their forties and fifties (Fig. 1A). Women made up $77 \%$ $(n=320)$ of the sample, and $23 \%(n=96)$ were men (Fig. 1B). The majority of the subjects were educated with college degrees and

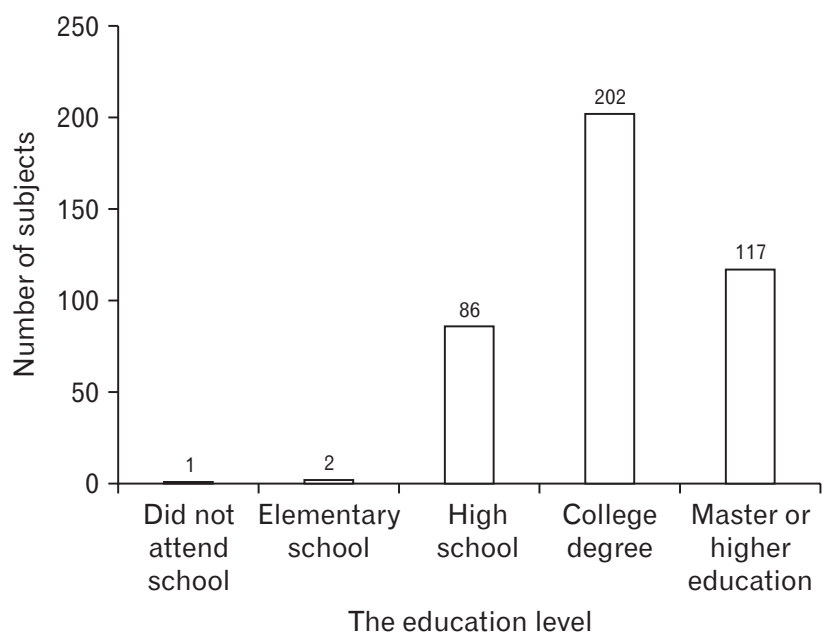

Figsure 2. The highest education level of our subjects.

with income of $\$ 25000-75000$ per household (Fig. 2). The majority $(53 \%)$ of the participants were married, $18 \%$ were divorced and $22 \%$ never married. $68 \%$ were white American, $15 \%$ were Asian American, 11\% Latino, and 9\% were African American or other races.

When subjects were asked about GI complaints, $54 \%$ had one or more GI symptoms, but only $22 \%$ ( $\mathrm{n}=91$ ) fulfilled the Rome IV criteria for IBS. IBS was significantly more prevalent in women $\chi^{2}(1)=18.89, P<0.001$. Among those 91 cases, 9 were male and 82 were female which corresponds to $9.4 \%$ of the males and $26 \%$ 
A

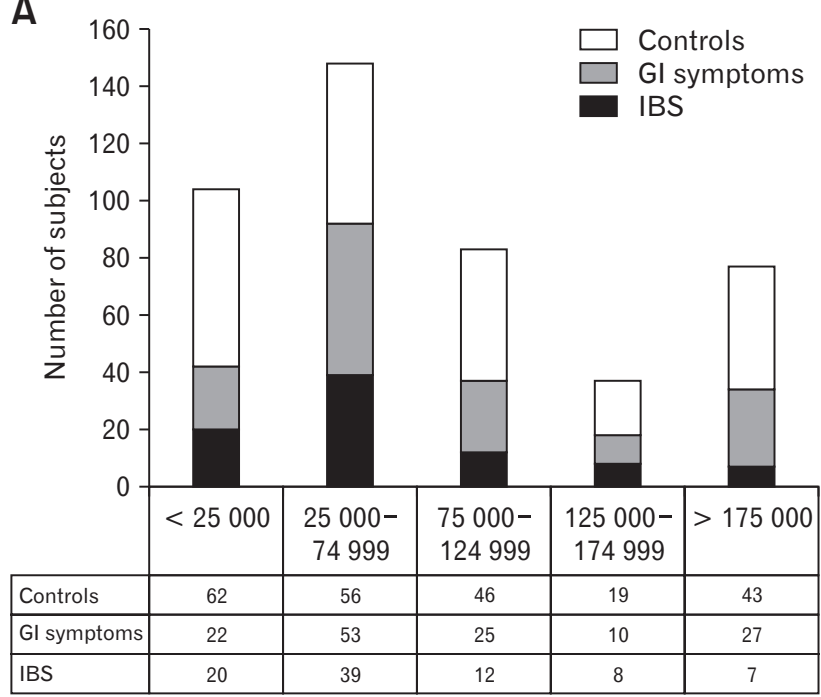

Annual income in $\$$
B

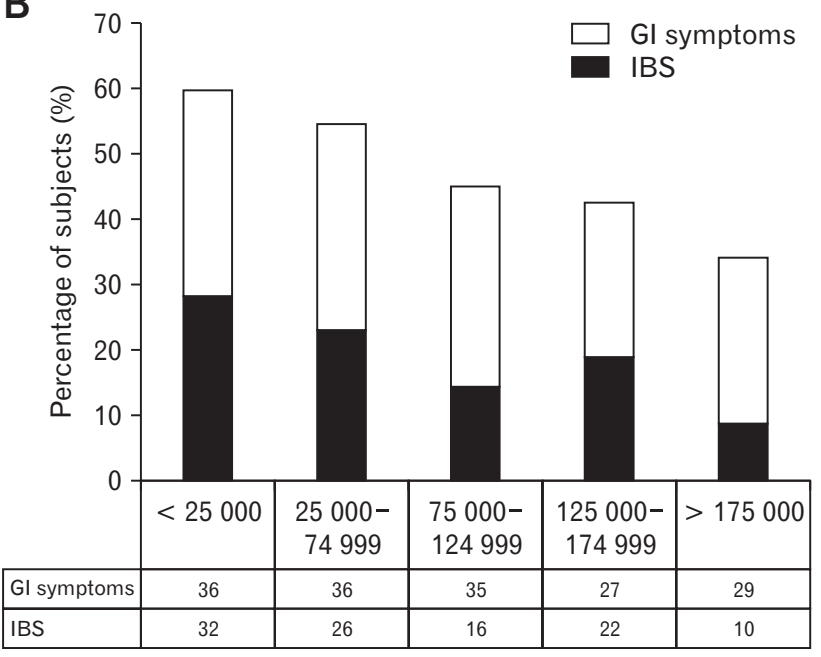

Annual income in \$

Figure 3. The frequency and prevalence of gastrointestinal (GI) symptoms and irritable bowel syndrome (IBS) in our subjects based on household income categories. (A) The frequency of GI symptoms and IBS in our subjects based on household income categories. (B) The prevalence of GI symptoms and IBS in our subjects based on household income categories.

of females who participated in our survey. GI symptoms were also significantly more prevalent in females $\chi^{2}(1)=11.84, P<0.001$. Among 225 subjects with GI symptoms, $15 \%$ were men and $85 \%$ were women, which corresponds to one-third of the male subjects and two-thirds of the female subjects who participated in our survey. Four percent of the study subjects had an active or inactive form of inflammatory bowel disease including ulcerative colitis and or Crohn's disease.

Fifty-four percent of the study subjects reported past or present anxiety but only $28 \%$ had been formally evaluated and/or treated for anxiety disorder in the past or at the time of the study. Among those who reported past or present anxiety, only $71 \%$ experienced anxiety symptoms in the last 2 weeks and were considered anxious based on the GAD-7 criteria. Twenty-nine percent had no or minimal anxiety symptoms in the last 2 weeks. Based on our data $40 \%$ of the men and $58 \%$ of women who participated in our survey had a history of anxiety disorder. Those with history of anxiety had significantly higher odd of having GI symptoms ( $68 \%$ vs $38 \%$ ), $\chi^{2}(1)$ $=29.52, P<0.001$ and IBS (33\% vs $10 \%)$ compared with those who did not have anxiety, $\chi^{2}(1)=35.40, P<0.001$.

Among the study subjects $52 \%$ reported past or present depression but only $29 \%$ had been formally evaluated and or treated for depression in the past or at the time of the study. Among those who reported past or present depression, only $69 \%$ experienced depressive symptoms in the last 2 weeks and were considered depressed

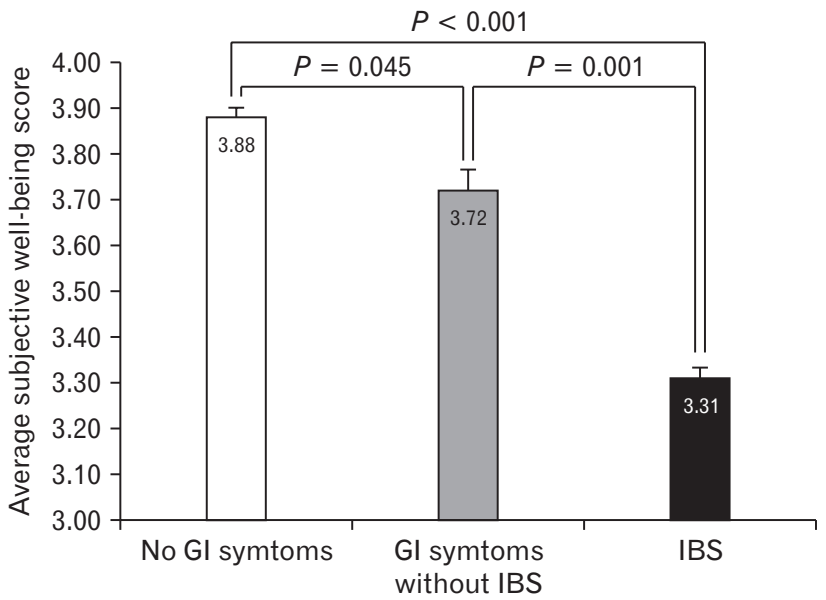

Figure 4. Average subjective well-being score in healthy control, those with gastrointestinal (GI) symptoms without irritable bowel syndrome (IBS) and those with IBS.

based on the PHQ-9 criteria. Thirty-one percent had no or minimal depressive symptoms in the last 2 weeks. Based on our data, $36 \%$ of the males and $54 \%$ of females who participated in our survey had a history of depression. Similarly, those with a history of depression had significantly higher prevalence of GI symptoms (69\% vs 39\%), $\chi^{2}(1)=37.70, \mathrm{P}<0.001$ and $\operatorname{IBS}(34 \%$ vs $11 \%)$, compared with those who did not have depression $\chi^{2}(1)=30.41, P<0.001$.

The level of household income was related to the presence of 


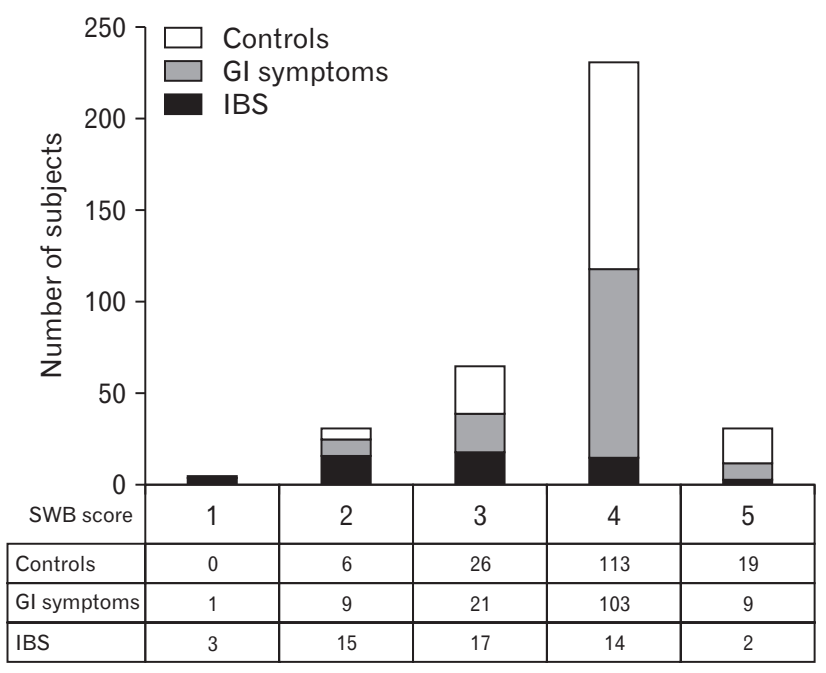

Figure 5. The frequency of gastrointestinal (GI) symptoms and irritable bowel syndrome (IBS) in our subjects based on subjective wellbeing (SWB) score categories.

GI symptoms and the prevalence of IBS. In general, those with GI symptoms or IBS were statistically more likely to have a lower income than the control group $(P=0.001$ and $P=0.002$, respectively, Mann-Whitney $U$ test). As we can see from Figures $3 \mathrm{~A}$ and $3 \mathrm{~B}$ there is a decrease in the likelihood of GI symptoms and IBS with an increase in household income. In addition, those who had GI symptoms had significantly less annual household income $(\mathrm{M}=\$ 92$ 000.00, $\mathrm{SD}=\$ 65500.00)$ compared to those who $\operatorname{did} \operatorname{not}(\mathrm{M}=$ $\$ 115500.00, \mathrm{SD}=\$ 69500.00), t(390)=3.45, P=0.001$. Similarly, those with IBS had significantly less annual household income $(\mathrm{M}=\$ 83750.00, \mathrm{SD}=\$ 62000.00)$ compared to those without IBS $(\mathrm{M}=\$ 107500.00, \mathrm{SD}=\$ 69000.00), t(390)=2.89, P=$ 0.004 .

We assessed subjective well-being using multiple questions exploring the well-being attributes. The average score of these attributes in healthy control and those with GI symptoms with and without IBS are shown in Table. Subjects with GI symptoms with or without IBS scored significantly lower in all of these well-being attributes.

The average subjective well-being score was lower in those with IBS compared to those with GI symptoms without IBS. People with GI symptoms, without IBS had lower average subjective wellbeing scores compared to those without GI symptoms using the non-parametric tests $(P<0.001$, Kruskal-Wallis test). Further subanalysis showed a stepwise decline in the average subjective wellbeing (Fig. 4). A similar stepwise decline were observed in the other well-being attributes, including sense of financial security $(P<$

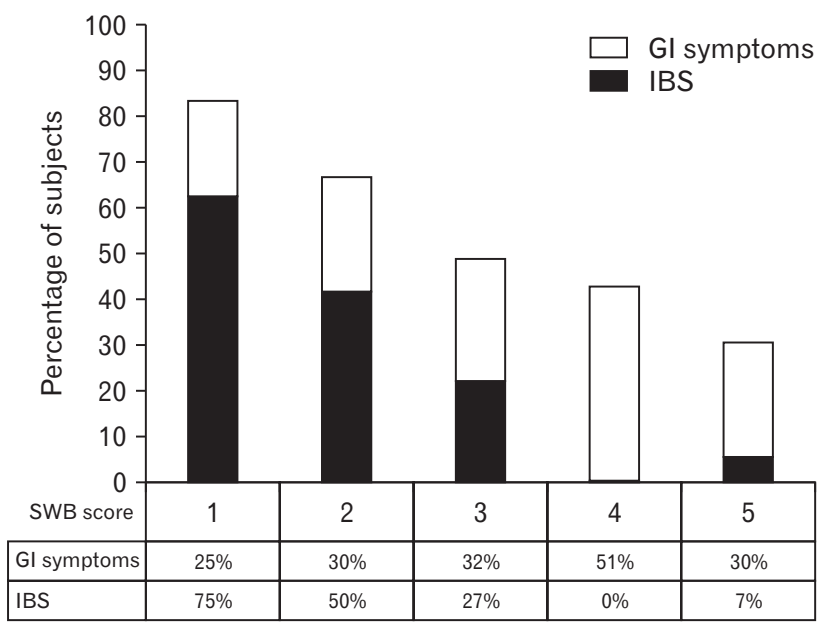

Figure 6. The prevalence of gastrointestinal (GI) symptoms and irritable bowel syndrome (IBS) in our subjects based on subjective wellbeing (SWB) score categories.

0.001), having a desirable job $(P<0.001)$, being respected $(P<$ $0.001)$, feeling their talent is being properly used $(P<0.001)$, having adequate pleasure in life $(P<0.002)$, feeling accepted by others $(P=0.001)$, living in peace $(P<0.001)$, being able to manage their life properly $(P<0.001)$, general sense of optimism $(P=$ $0.017)$, believe in fairness in life $(P=0.017)$, and being successful $(P<0.001)$ (Table).

As depicted in in Figures 5 and 6, the prevalence of IBS closely follows the overall sense of subjective well-being for our data. After stratification for anxiety, in those with or without history of current or past anxiety, subjective well-being was still a significant predictor of the presence of IBS ( $P=0.004$ in those without anxiety and $P=0.011$ in those with anxiety, Binary logistic regression). After stratification for depression, in those with or without history of current or past depression, subjective well-being was still a significant predictor of the presence of IBS $(P=0.044$ in those without depression and $P=0.006$ in those with depression, Binary logistic regression). Furthermore, when we used 4 major predictors in a multivariate model using Binary logistic regression, we observed that only subjective well-being and presence of anxiety remained a reliable predictor of the presence of IBS while both presence of depression and household income no longer proved to be a predictor in this model.

\section{Discussion}

GI symptoms are very common complaints in the general population, but only a subgroup of these individuals could be 
characterized as experiencing IBS. IBS is a functional GI disorder associated with a significant negative impact on individual personal, social, and financial aspects of life, and results in significant decline in quality of life. Several studies have demonstrated the correlation of commonly diagnosed psychological conditions, such as anxiety and depression, with FGID and IBS. ${ }^{11-16}$ Those also established the presence of several negative well-being attributes in individuals with IBS. ${ }^{17-19}$ Knowing the specifics of the affected attributes of wellbeing could open a new horizon for helping these individuals, using specific forms of psychosocial interventions. ${ }^{27-33}$ When we can tailor these interventions based on the affected well-being attributes, and the effect of these interventions may be improved.

Several of these well-being attributes contribute to our sense of subjective well-being and overall contentment with life. In this study we investigated the correlation of several well-being attributes and sense of subjective well-being and determined whether these attributes correlated with the presence or absence of GI symptoms and or IBS. Since the majority of research has historically focused on the role of psychopathology in IBS, we investigated whether subjective well-being correlated with IBS above and beyond the known psychopathology model. Our data suggest that positive psychological principles correlate with GI symptoms and IBS, independent of the presence of common psychological conditions such as anxiety and depression.

The prevalence of IBS in our study population was around $22 \%$, which is on high end of previously reported data. There could be several reasons for the high prevalence of IBS in our study population. One reason could be the preponderance of young female subjects in our study, which inherently have a higher prevalence of IBS. $^{34}$ As expected, the prevalence of IBS was significantly higher in women compared to men (3:1). Moreover, there may be a selfselection artifact or ascertainment issue since those with IBS may be more interested in research in IBS and therefore, be more willing to participate in the survey. More than half (54\%) of our study subjects suffered from some form of GI symptoms, but only one third of the individuals (38\%) met the criteria to be categorized as having IBS.

Among the factors investigated, the level of household income negatively related to the presence of GI symptoms and IBS. This was similar to the large US household survey of functional bowel disease. ${ }^{34}$ Other studies showed that there is no relationship between household income and IBS prevalence, and one study showed an increase rate of IBS in university students and those with higher household income. ${ }^{35,36}$ The connection of household income and IBS could also be through its interconnection with well-being and
IBS, since several studies showed that the level of happiness and household income have a positive correlation. ${ }^{37,38}$

Our data showed that there was a significant correlation of subjective well-being, several well-being attributes, and the presence of GI symptoms and IBS. In particular, we showed that the sense of confidence, sense of financial security, feeling loved, feeling healthy and fit, having a passion in life, having a desirable job, sense of independence, being respected, feeling their talent is being properly used, having adequate pleasure in life, feeling accepted by others, living in peace, being able to manage their life properly, general sense of optimism, sense of being successful, sense of excess regret from the past actions, and overall sense of happiness negatively correlated with GI symptoms and/or IBS (Table). We should consider that the direction of the relationship of these well-being attributes with IBS is not specified, and those attributes could have been negatively affected by a chronic recurring illness such as IBS and its recurrent nuisance symptoms. For example, even contemplating the symptoms can erode the sense of confidence, feeling of being healthy and fit, sense of living in peace, being able to manage their life and general sense of optimism. However, it is harder to justify the significant correlation of other variables solely on the presence of IBS such as not feeling loved enough, not being respected or accepted by others, not having a passion in life, not feeling that their talent is being properly used, reduced trust in others, and sense of excess regret from the past. Our data is exploratory in nature and our results may propose that well-being attributes may have a contributory effect in the pathogenesis of the GI symptoms and/or IBS through a biopsychosocial model. However, larger studies may be needed to confirm this preliminary information.

There are many studies that showed trends in particular wellbeing attributes in IBS and correlation with IBS severity. We only limit our discussion to a few. For example, our finding of lower confidence and feeling of being accepted by others in those with GI symptoms. The subjects with IBS had feelings of being less accepted, but their lower level of confidence showed a negative trend but did not reach statistical significance. This finding is similar to the finding of Grodzinsky who documented low self-esteem among subjects with IBS. ${ }^{39}$ As part of well-being attributes we asked our study subjects a question evaluating the ability to forgive and forget mistakes. Mazaheri et al showed that attributes such as forgiveness were protective factors for emotional dysregulation and IBS. ${ }^{18} \mathrm{In}$ our study, we did not find any significant difference in this ability to forgive and or forget in those with GI symptoms and/or IBS compared to our controls. Similar to the ability to forgive and forget mistakes, flexibility was another important attribute that contributes 
to coping mechanism. Marianda et al showed that "catastrophizing" (a maladaptive way of coping) was directly associated with IBS severity. ${ }^{17}$ Other studies also showed that coping is an important defense mechanism that is negatively affected in those with IBS. ${ }^{39,40}$

Not all the well-being attributes that are considered to be associated with general well-being showed a significant correlation with GI symptoms or IBS. In particular, we did not find any significant correlation with having someone to love, belief and practice of a particular faith, perceived generosity, amount of being supportive to others, the satisfaction with the adequateness of formal education, having a regular leisure program, self-recognition of attractiveness, general sense of trusting others, feeling grateful for what we have, and finally feeling closure with death.

Our data showed that the prevalence of IBS closely follows the overall sense of subjective well-being. However, the question arises whether this variable is a predictor of the GI symptoms and IBS independent of known variables such as presence of anxiety and depression. It is believed that anxiety and depression could be the main form of psychological issues in those with GI symptoms, and particularly $\mathrm{IBS}^{41}$ Our data, however, showed that after stratification for anxiety and depression, there was still a significant correlation of sense of subjective well-being and GI symptoms and presence of IBS. This demonstrated that factors involved in a perceived sense of well-being could contribute or correlate with presence of GI symptoms and IBS, independent of anxiety and or depression. Considering the high prevalence of psychiatric co-morbid conditions in patients with IBS who are referred to tertiary care centers and the positive effect of behavioral therapies for IBS, it is likely that tailoring our therapy toward the issues that are more directly aimed at these specific problems, particularly those that can directly or indirectly affect our well-being could yield more efficiency in our management. $^{42}$

The questionnaire was designed by our team for the purpose of evaluating subjective well-being and several well-being attributes. Even though we had good internal consistency in the responses, our study was a pilot study and we do not have a full validation of our well-being questionnaire. We will be able to fully validate the questionnaire only in the future studies with larger subset of subjects.

Our study had several shortcomings. Our study is exploratory in nature, and we use statistical evaluation of a large numbers of variables. This can result in inflation in the type I error rate for our tests, giving spurious significant results. The other shortcoming may be related to the design of our study, which inherently targeted educated individuals due to nature of an online survey. We also had a disproportionate number of females in our study, which again is another pitfall of online surveys. This is particularly true since higher prevalence of IBS in female subjects. This could have biased our data toward showing more significant correlations that we would have naturally had if we would have obtained equal numbers of men and women in our study. Due to the small number of participants, the study may have been underpowered to test various hypotheses among sub-groups of interests. This is particularly true regarding the small subgroups of IBS such as diarrhea predominant IBS, constipation predominant IBS, and alternating or mixed type IBS.

We had more than 80 questions in our questionnaire and some of those questions were deeply private for some individuals. We were not surprised that there were several well-being attribute questions that were left unanswered (up to $14 \%$ ) due to the long nature of the questionnaire or the private nature of the question. This was despite our effort to minimize asking too many detailed questions, to increase the chance of having a complete response. Regardless, we did not delete the cases that had some missed questions, but rather treated those variables with proper handling of missing data during particular analysis. However, partially filled questionnaires or missing data could have made our interpretation less effective. For this reason, we forfeited collecting more detailed information about GI symptoms that could have been useful in our interpretation.

Another issue that is also inherent to most survey researches is response bias. Even though the questionnaire was completely anonymous, it is possible that the response of the individuals may have been biased toward answering what they thought should be the right answer rather than how they could have really scored themselves. Selection bias is another issue that can affect survey research. The population that was selected for our study was limited to whom used social media, and it may not represent a general population. Not to mention that, it is likely that those with GI symptoms and/ or IBS are more willing to participate in such a long survey, and artificially increase the prevalence of GI symptoms and IBS. Other investigators have also shown that the frequency or severity of the IBS can be overestimated using surveys due to ascertainment bias. ${ }^{43}$ Overall, we consider this study as a preliminary exploratory study to help us to design a more comprehensive study with a larger number of participants to examine this very interesting subject.

\section{Conclusion}

There is a clear correlation between commonly diagnosed psychological conditions such as anxiety and depression in FGID and IBS. In this study we showed that subjective well-being as well as several well-being attributes that contribute to our sense of 
overall contentment in life are negatively affected in those with GI symptoms or IBS. In addition, household income negatively correlated with the prevalence of GI symptoms and IBS. In particular, we showed that the subjective well-being and happiness correlated with the presence of IBS independent of anxiety and depression. It would be plausible to postulate that these variables could have a contributory effect in the pathogenesis of the GI symptoms and/or IBS through a biopsychosocial model.

Acknowledgements: We wish to thank Dr Douglass A Drossman who provided us with invaluable advice on our research and feedback in preparation of this manuscript.

\section{Financial support: None.}

\section{Conflicts of interest: None.}

Author contributions: Ashkan Farhadi, Dwaine Banton, and Laurie Keefer were involved in study concept and design, acquisition of data, analysis and interpretation of data, and drafting of the manuscript and critical revision of the manuscript for important intellectual content.

\section{References}

1. Hungin AP, Whorewell PJ, Tack J, Mearin F. The prevalence, patterns and impact of irritable bowel syndrome: An international survey of 40 000 subjects. Aliment Pharmacol Ther 2003;17:643-650.

2. Farhadi A, Bruninga K, Fields J, Keshavarzian A. Irritable bowel syndrome: an update on therapeutic modalities. Expert Opin Investig Drugs 2001;10:1211-1122.

3. Tanaka K, Kanazawa M, Fukudo, drossman DA. Biopsychosocial Model of Irritable Bowel Syndrome. J Neurogastroenterol Motil 2011;17:131-139.

4. Lee SP, Sung IK, Kim JH, Lee SY, Park HS, Shim CS. The Effect of Emotional Stress and Depression on the Prevalence of Digestive Diseases. J Neurogastroenterol Motil 2015;21:273-282.

5. Muscatello MR, Bruno A, MentoC, Pandolfo G, Zoccali RA. Personality traits and emotional patterns in irritable bowel syndrome. World J Gastroenterol 2016;22:6402-6415.

6. Bennett EJ, Piesse C, Palmer K C, Tennant C, Kellow JE. Functional gastrointestinal disorders: psychological, social, and somatic features. Gut 1998;42:414420.

7. Azpiroz F, Guyonnet D, Donazzolo Y, Gendre D, Tanguy J, Guarner F. Digestive symptoms in healthy people and subjects with irritable bowel syndrome; validation of symptom frequency questionnaire. Clin Gastroenterol 2015;49:64-70.

8. Walter A, Jones MP, Talley NJ, Kjellstrom L, et al. Abdominal pain is associated with anxiety and depression scores in a sample of the general adult population with no signs of organic gastrointestinal disease. Neurogastroenterol Motil 2013;25:741-750.

9. Ben-Ezra M, Hamama-Raz Y, Palgi S, Palgi Y. Cognitive appraisal and psychological distress among patients with irritable bowel syndrome. Isr J Psychiatry Relat Sci 2015;52:54-59.

10. Drossman DA, Hasler WA. Rome IV--functional GI disorders: disorders of gut-brain interaction. Gastroenterology 2016;150:1257-1261.

11. Roohafza H, Bidaki EZ, Hasanzadeh-Keshteli A, Daghaghzade H, Afshar H, Adibi P. Anxiety, depression and distress among irritable bowel syndrome and their subtypes: an epidemiological population based study. Adv Biomed Res 2016;5:183.

12. Popa SL, Dumitrascu DL. Anxiety and IBS revisited: ten years later. Clujul Medical 2015;88:253-257.

13. MykletunA, Jacka F, Williams L, et al. Prevalence of mood and anxiety disorder in self reported irritable bowel syndrome (IBS). An epidemiological population based study of women. BMC Gastroenterol 2010;10:88.

14. Lee S, Wu J, Ma YL, Tsang A, Guo J, Sung J. Irritable bowel syndrome is strongly associated with generalized anxiety disorder: a community study. Aliment Pharmacol Ther 2009;30:643-651.

15. Grzesiak1 M, Beszłej JA, Mulak A et al. The lifetime prevalence of anxiety disorders among patients with irritable bowel syndrome. Adv Clin Exp Med 2014;23:987-992.

16. Ladep NG, Obindo TJ, Audu MD, Okeke EN, Malu AO. Depression in patients with irritable bowel syndrome in Jos, Nigeria. World J Gastroenterol 2006;12:7844-7847.

17. van Tilburg MA, Palsson OA, Whitehead WE. Which psychological factors exacerbate irritable bowel syndrome? development of a comprehensive model. Psychosom Res 2013;74:486-492.

18. Mazaheri M, Nikneshan S, daghaghzadeh $\mathrm{H}$, Afshar $\mathrm{H}$. The role of positive personality traits in emotion regulation of patients with irritable bowel syndrome (IBS). Iran J Public Health 2015;44:561-569.

19. Farnam A, Somi MH, Sarami F, Farhang S, YasebiniaS. Personality factors and profiles in variants of irritable bowel syndrome. World J Gastroenterol 2007;13:6414-6418.

20. Krarup AL, Peterson E, Ringström G, Tornblom H, Hjortswang H, Simren M. The short health scale: a simple, valid, reliable, and responsive way of measuring subjective health in patients with irritable bowel syndrome. J Clin Gastroenterol 2015;49:565-570.

21. Andrae DA, Patrick DL, Drossman DA, Covington P. Evaluation of the irritable bowel syndrome quality of life (IBS-QOL) questionnaire in diarrheal-predominant irritable bowel syndrome patients. Health and Qual Life Outcomes 2013;11:208.

22. Jamali R, Raisi M, Matini M, Moravveji A, Omidi A, Amini J. Health related quality of life in irritable bowel syndrome patients, Kashan, Iran: A case control study. Adv Biomed Res 2015;4:75.

23. Simrén M, Abrahamsson H, Svedlund J, Bjornsson ES. Quality of life in patients with irritable bowel syndrome seen in referral centers versus primary care: the impact of gender and predominant bowel pattern. Scand J Gastroenterol 2001;36:545-552.

24. DienerE, Tay L. Subjective well-being and human welfare around the 
world as reflected in the Gallup World Poll. Int J Psychol. 2015;50:13549.

25. Zautra A, Hempel A. Subjective well-being and physical health/ a narrative literature review with suggestions for future research. Int J Aging Hum Dev 1984;19:91-110.

26. Roth B, Ohlsson B. Gastrointestinal symptoms and psychological well-being in patients with microscopic colitis. Scand J Gastroenterol 2013;48:27-34.

27. Ballou S, Keefer L. Psychological interventions for irritable bowel syndrome and inflammatory bowel diseases. Clin Transl Gastroenterol 2017;8:214.

28. Guthrie E, Creed F, Dawson D, Tomenson B. A randomised controlled trial of psychotherapy in patients with refractory irritable bowel syndrome. Br J Psychiatry 1993;163:315-321.

29. Hauser G, Pletikosic S, Tkalcic M. Cognitive behavioral approach to understanding irritable bowel syndrome. World J Gastroenterol 2014; 20:6744-6758.

30. Houghton LA, Calvert EL, Jackson NA, Cooper P, WhorwellPJ. Visceral sensation and emotion: a study using hypnosis. Gut 2002;51:701704.

31. Hunt MG, Moshier S, Milonova M. 30 Brief cognitive-behavioral internet therapy for irritable bowel syndrome. Behav Res Ther 2009; 47:797-802.

32. Tonkin-Crine1 S, Bishop FL, Ellis M, Moss-morris R, Everitt H. Exploring patients' views of a cognitive behavioral therapy-based website for the self-management of irritable bowel syndrome symptoms. J Med Internet Res 2013;15:190

33. Zijdenbos IL, de Wit NJ, van der Heijden GJ, Rubin G, Quartero AO. Psychological treatments for the management of irritable bowel syndrome (Review). Cohrane Database Syst Rev 2009;1: 2.

34. Drossman DA, Li Z, Andruzzi E, et al. U.S. householder survey of functional gastrointestinal disorders. Prevalence, sociodemography, and health impact. Dig Dis Sci 1993;38:1569-1580.

35. Minocha A, Johnson WD, Abell TL, Wigington WC. Prevalence, sociodemography, and quality of life of older versus younger patients with irritable bowel syndrome: a population-based study. Dig Dis Sci 2006;51:446-453.

36. Costanian C, Tamim H, Assaad SK. Prevalence and factors associated with irritable bowel syndrome among university students in Lebanon: Findings from a cross-sectional study. World J Gastroenterol 2015;21:3628-3635

37. Stevenson B, Wolfers J. Economic growth and subjective well-being: Researching the Easternlin paradox. National Bureau Econ Res 2008;39:1102 .

38. Helliwell J, Layard R, Sachs J. World happiness report. New York: The Earth Institute, Columbia University 2012.

39. Grodzinsky E, Walter S, Viktorsson L, Carlsson AK, Jones MP, Faresjo A. More negative self-esteem and inferior coping strategies among patients diagnosed with IBS compared with patients without IBS - a casecontrol study in primary care. BMC Fam Pract 2015;16:6.

40. Stanculete MF, Matu S, Pojoga C, Dumitrascu DL. Coping strategies and irrational beliefs as mediators of the health-related quality of life impairments in irritable bowel syndrome. J Gastrointestin Liver Dis 2015;24:159-164.

41. Koloski NA, Talley NJ, Boyce PM. Does psychological distress modulate functional gastrointestinal symptoms and health care seeking? A prospective, community Cohort study. Am J Gastroenterol 2003;98:789797.

42. Park SH, Videlock EJ, Shih W, Presson AP, Mayer EA, Chang L. Adverse childhood experiences are associated with irritable bowel syndrome and gastrointestinal symptom severity. Neurogastroenterol Motil 2016;28:1252-1260.

43. Drossman DA. Abuse, trauma, and GI illness: is there a link? Am J Gastroenterol 2011;106:14-25. 